\title{
Flavonoid Tablet
}

National Cancer Institute

\section{Source}

National Cancer Institute. Flavonoid Tablet. NCI Thesaurus. Code C74030.

A tablet formulation of flavonoids with antioxidant and potential chemopreventive activities. Polyphenolic, soluble plant pigment flavonoids inactivate oxygen radicals, prevent lipid peroxidation, and inhibit DNA oxidation. In vitro, these agents have been shown to increase the rate of apoptosis, and inhibit cell proliferation and angiogenesis.

Furthermore, flavonoids can induce conjug ating enzymes, such as glutathione transferases and glucuronosyltransferases. 